\title{
THE PECULIAR SYMBIOTIC STAR CH CYG - A BRIGHT COUNTERPART OF CATACLYSMIC VARIABLES?
}

\author{
LAURITS LEEDJÄRV \\ Tartu Observatory \\ EE 2444 Tôravere, Estonia
}

\section{Introduction}

CH Cyg has begun to show symbiotic features only since 1963. Up to now four outbursts of $\mathrm{CH}$ Cyg have been recorded, the fifth going on at the present time. CH Cyg reveals a lot of peculiarities as compared to 'normal' symbiotic stars (extended inactive periods between the 'symbiotic' periods; the most extended active period in 1977-1986 including the ejection of bipolar radio jets; a stage of increased activity since 1992, with peculiar profiles of the Balmer emission lines etc.); see Mikołajewski, Mikołajewska \& Khudyakova (1990) for further references.

Classical models for explaining the outbursts of symbiotic stars (e.g. Mikołajewska \& Kenyon 1992) are unable to account for the behaviour of $\mathrm{CH}$ Cyg. The magnetic rotator model has been proposed and developed by Mikołajewski \& Mikołajewska $(1988,1996)$. The model includes a wind accreting, magnetic $\left(\mathrm{B} \sim 10^{7} \mathrm{G}\right)$, rapidly rotating $(\mathrm{P} \sim 8 \mathrm{~m})$ white dwarf (WD) as the hot component of the symbiotic system. A strong magnetic field prevents matter from the red giant's wind being accreted onto the WD surface in the usual way. Instead, three stages of accretion could be considered: inactive, propeller and accretor state.

\section{Recent manifestations of the propeller action}

The latest active period of CH Cyg observed since early 1992 is characterized by a new type of peculiarity: high-velocity (up to $\gtrsim 1000 \mathrm{~km} \mathrm{~s}^{-1}$ ) blueand red-shifted broad emission components of Balmer lines. Examples of the spectra of CH Cyg obtained during 1992-1994 with the $1.5 \mathrm{~m}$ telescope at the Tartu Observatory have been published by Leedjärv \& Mikołajewski (1995). 
The additional emission (occasionally also absorption) components are variable on a time-scale of days and, as shown by Tomov et al. (1995), even hours and minutes. Most of the time only blue-shifted features are visible. The magnetic rotator model is as follows. Matter accreted by the WD is expelled back by the rapidly rotating magnetosphere of the WD. This outward flow of matter is not quite continuous, instead we can see individual blobs of matter, rejected by the propeller. Most of the rejected blobs should be expelled towards us, and the blue-shifted emission and absorption features are visible. Possible flip-flop instabilities of the accretion wake (e.g. Livio et al. 1991) on a time-scale of weeks or months can produce blobs which could reach the magnetosphere on the receeding hemisphere of the propeller, causing red-shifted emission components (without absorption) occasionally.

During active periods brightness variations of $\mathrm{CH}$ Cyg on a time-scale of minutes can be observed, especially at shorter wavelengths. This kind of flickering is common for many types of cataclysmic variables, including the recurrent novae T CrB and RS Oph. During the present propeller state for example, Tomov et al. (1996) have announced flickering with amplitude $\sim 0.7 \mathrm{mag}$ in $B$ light in 1994 June. We observed CH Cyg during several nights in 1995 May with the $60 \mathrm{~cm}$ telescope and the double-beam, skychopping photometer at the Tuorla Observatory, Finland. In spite of the low brightness level of $\mathrm{CH}$ Cyg $(V \approx 9 \mathrm{mag}$ ), flickering is still present, reaching amplitude $\sim 0.6 \mathrm{mag}$ in $U$ and $\sim 0.4 \mathrm{mag}$ in $V$.

Emission line profiles of $\mathrm{CH}$ Cyg observed since 1992 are quite unique among variable stars. Only the recurrent nova RS Oph has demonstrated similar features (Lijima et al. 1994). On the other hand, the propeller phenomenon considered mostly in the case of neutron stars, could be more widespread among magnetic white dwarfs. $\mathrm{CH}$ Cyg as a relatively bright star $(V \approx 6-9 \mathrm{mag}$ ) could serve as a testing site of interactions between matter and magnetic field, relevant to many types of much fainter cataclysmic variables.

\section{References}

Iijima, T., Strafella, F., Sabaddin, F., Bianchini, A., 1994, A\&A, 283, 919

Leedjärv, L., Mikołajewski, M., 1995, A\&A, 300, 189

Livio, M., Soker, N., Matsuda, T., Anzer, U., 1991, MNRAS, 253, 633

Mikołajewska, J., Kenyon, S.J., 1992, MNRAS, 256, 177

Mikolajewski, M., Mikolajewska, J., 1988, in "The Symbiotic Phenomenon", Mikolajewska J. et al. (eds.), Kluwer, p233

Mikołajewski, M., Mikołajewska, J., 1996, MNRAS, submitted

Mikołajewski, M., Mikołajewska, J., Khudyakova, T.N., 1990, A\&A, 235, 219

Tomov, T., Kolev, D., Munari, U., Antov, A., 1996, MNRAS, submitted 